\title{
LOCALIZED DENSITY MATRIX MINIMIZATION AND LINEAR-SCALING ALGORITHMS
}

\author{
RONGJIE LAI AND JIANFENG LU
}

\begin{abstract}
We propose a convex variational approach to compute localized density matrices for both zero temperature and finite temperature cases, by adding an entrywise $\ell_{1}$ regularization to the free energy of the quantum system. Based on the fact that the density matrix decays exponentially away from the diagonal for insulating systems or systems at finite temperature, the proposed $\ell_{1}$ regularized variational method provides an effective way to approximate the original quantum system. We provide theoretical analysis of the approximation behavior and also design convergence guaranteed numerical algorithms based on Bregman iteration. More importantly, the $\ell_{1}$ regularized system naturally leads to localized density matrices with banded structure, which enables us to develop approximating algorithms to find the localized density matrices with computation cost linearly dependent on the problem size.
\end{abstract}

\section{INTRODUCTION}

Efficient calculation of the low-lying spectrum of operators plays a central role in many applications. In particular, in the context of electronic structure theory, given a discretized effective Hamiltonian (such as the current iterate in a self-consistent iteration), the goal is to obtain the density matrix corresponding to the number of electrons. For zero temperature, the density matrix is the projection operator onto the low-lying eigenspace; for finite temperature, the density matrix is given by the Fermi-Dirac function acting on the Hamiltonian 19.28.

In this work, we extend the variational approach for localized density matrix in our previous work [16 to finite temperature, by adding entrywise $\ell_{1}$ penalty to the free energy of the quantum system (which, in the context of density functional theory, corresponds to the linear version of the Mermin functional [22]). We also theoretically show that the proposed localized density matrix approximates, via the Frobenius norm, the true density matrix linearly dependent on the regularization parameter $1 / \eta$. In addition, convergence guaranteed numerical algorithms are also designed to solve the proposed problems based on Bregman iteration.

Date: October 12, 2015.

The research of R.L. is partially supported by the National Science Foundation under award DMS1522645. The research of J.L. was supported in part by the Alfred P. Sloan Foundation and the National Science Foundation under award DMS-1312659 and DMS-1454939. The authors like to thank Stanley Osher and Vidvudz Ozolins for their encouragements and helpful discussions. 
More importantly, this paper focuses on efficient algorithms to minimize the variational problem for localized density matrices both at zero and finite temperature. In particular, we develop linear-scaling algorithms such that the computational cost scales linearly with the dimension of the matrix. The key idea is to exploit the decay of the density matrix away from the diagonal. The $\ell_{1}$ regularized localized density matrices enable us to approximate the original variational problem by restricting to banded matrices.

Linear-scaling algorithms have been an important research direction in electronic structure calculation since the 1990s. Closely related to our context is the density matrix minimization (DMM) algorithms, which is first introduced by Li, Nunes, and Vanderbilt [17] and Daw [6], and have been further developed since then, see e.g., the reviews 3. 11. These algorithms are based on the fact that for insulating system or systems at finite temperature, the density matrix decays exponentially away from the diagonal (see e.g., [1, 7, 15]). Thus, one may require the density matrices to satisfy prescribed sparsity structures, such as banded matrices for 1D problem. As a result, the degree of freedom and the computational cost becomes linearly scaling. Another closely related class of methods is the purification method of density matrix (see e.g., [20 21 23 27] and a review [24]). Unlike the approach of DMM and the method we take in this work, these methods are not variational.

We emphasize a crucial difference between our approach and the previous works: Our variational problem is still convex even after truncation! This is in stark contrast to the previous formulations where the convexity is lost by using purification [21] or other techniques to approximate the inverse of density matrix [4]. This loss of convexity often introduces local minimizers to the variational problem and also issues of convergence. We note that even when the $\ell_{1}$ regularization is dropped from our variational problem, it is still convex and different from the standard DMM algorithms. In fact, it would be of interest to explore this convex formulation, which will be considered in our future work.

The rest of the paper is organized as follows. In the next section, we introduce the variational principles for localized density matrices for both zero and finite temperature cases, with their approximation properties. In Section 3 we introduce the Bregman iteration type algorithms to solve these minimization problems. linear-scaling algorithms are discussed in Section 4 . We validate the algorithms through numerical examples in Section 5. Some conclusive remarks are discussed in Section 6,

\section{LOCALIZED DENSITY MATRIX MINIMIZATION}

In this work, we will consider a family of energy functionals with parameters $\beta$ and $\eta$ :

$$
\mathscr{E}_{\beta, \eta}=\operatorname{tr}(H P)+\frac{1}{\beta} \operatorname{tr}\{P \ln P+(1-P) \ln (1-P)\}+\frac{1}{\eta}\|P\|_{1}
$$

where $\|\cdot\| \|$ denotes the entrywise $\ell_{1}$ norm of a matrix and $H$ and $P$ are $n \times n$ symmetric matrices, which are respectively the (discrete) Hamiltonian and density matrix in the context of electronic structure calculation. Here, $\beta$ is the inverse temperature and $\eta$ is 
the parameter for the $\ell_{1}$ regularization. The functional $\mathscr{E}_{\beta, \eta}$ and its terms will be further explained below.

2.1. Background of density matrix minimization. Let us recall the starting point of [16], which is also the base of the functional $E_{\beta, \eta}$, is the convex variational principle for density matrix

$$
\begin{aligned}
& \min _{P \in \mathbb{R}^{n \times n}} \mathscr{E}_{\infty, \infty}(P)=\min _{P \in \mathbb{R}^{n \times n}} \operatorname{tr}(H P), \\
& \text { s.t. } \operatorname{tr} P=N, P=P^{\mathrm{T}}, 0 \leq P \leq I,
\end{aligned}
$$

where the notation $A \leq B$ denotes that $B-A$ is a symmetric positive semi-definite matrix and $N$ denotes the number of electrons. Note that the constraint $0 \leq P \leq I$ is the convexification of the idempotency constraint $P=P^{2}$, which provides the same minimizer for non-degenerate problems (see e.g., [16. Proposition 1]). Indeed, denote $\left\{\lambda_{i}, \phi_{i}\right\}_{i=1}^{n}$ the eigenvalue and eigenvector pairs of $H$ with the assumption $\lambda_{N}<\lambda_{N+1}$, the solution of 2) is given by

$$
P_{\infty, \infty}=\sum_{i=1}^{N} \phi_{i} \phi_{i}^{\mathrm{T}}
$$

the projection operator on the subspace spanned by the first $N$ eigenvectors, which is known as the density matrix in physics terms.

The variational principle (2) corresponds to the physical zero temperature case (and hence the inverse temperature $\beta=\infty$ ). At zero temperature, the electrons prefer to occupy lowest possible energy states with the restriction of Pauli's exclusion principle that a single state can be occupied by only one electron (we ignore spin degeneracy). Therefore, the density matrix corresponds to the projection operator to the first $N$ eigenvectors. This is no longer the case at finite temperature. For finite temperature, the system minimizes the free energy, which is the sum of the energy and entropy contribution:

$$
\begin{aligned}
& \min _{P \in \mathbb{R}^{n \times n}} \mathscr{E}_{\beta, \infty}(P)=\min _{P \in \mathbb{R}^{n \times n}} \operatorname{tr}(H P)+\frac{1}{\beta} \operatorname{tr}\{P \ln P+(1-P) \ln (1-P)\} \\
& \text { s.t. } \operatorname{tr} P=N, P=P^{\mathrm{T}}, 0 \leq P \leq I,
\end{aligned}
$$

where we have used the Fermi-Dirac entropy

$$
\varphi(x)=x \ln x+(1-x) \ln (1-x), \quad x \in[0,1] .
$$

This particular entropy function defined on $[0,1]$ gives arise the familiar Fermi-Dirac distribution in quantum mechanics as we will explain now. First note that

$$
\begin{aligned}
& \varphi^{\prime}(x)=\ln x-\ln (1-x), \\
& \varphi^{\prime \prime}(x)=x^{-1}+(1-x)^{-1},
\end{aligned}
$$

and hence $\varphi^{\prime \prime}(x) \geq 4$ for $x \in[0,1]$. Therefore, $\operatorname{tr} \varphi(P)$ is strictly convex with respect to $P$ and the minimizer of 4 exists. To obtain the form of the minimizer, we write the 
Eular-Lagrange equation, given by

$$
H+\frac{1}{\beta}(\ln P-\ln (1-P))-\mu=0,
$$

where $\mu$ is the Lagrange multiplier for the constraint $\operatorname{tr} P=N$, known as the chemical potential. Solving the equation, we find that the unique minimizer to 4 is given by

$$
P_{\beta, \infty}=[1+\exp (\beta(H-\mu))]^{-1}=\sum_{i=1}^{n} \rho_{i} \phi_{i} \phi_{i}^{\mathrm{T}},
$$

where the second equality comes from the spectral decomposition of the matrix $H$, with $\rho_{i}$ being the occupation number of the $i$-th eigenstate, given by

$$
\rho_{i}=\frac{1}{1+\exp \left(\beta\left(\lambda_{i}-\mu\right)\right)} \in[0,1], \quad i=1, \ldots, n .
$$

Thus the electrons occupy the energy level according to the Fermi-Dirac distribution for finite temparature (see e.g., [19|). Requiring $\operatorname{tr} P_{\beta, \infty}=N$, the chemical potential $\mu$ is determined by

$$
\sum_{i=1}^{n} \frac{1}{1+\exp \left(\beta\left(\lambda_{i}-\mu\right)\right)}=N
$$

For a fixed Hamiltonian matrix, $\mu$ is then a function of $\beta$. It is not difficult to see that

$$
\lim _{\beta \rightarrow \infty} \mu(\beta)=\frac{1}{2}\left(\lambda_{N}+\lambda_{N+1}\right),
$$

which lies in between the highest occupied and lowest unoccupied eigenvalues.

2.2. Localized density matrix minimization. In [16], the following $\ell_{1}$ regularized version of the variational principle (2) is proposed as a convexified model for compressed modes introduced in [26].

$$
\begin{gathered}
P_{\infty, \eta}=\arg \min _{P \in \mathbb{R}^{n \times n}} \mathscr{E}_{\infty, \eta}=\min _{P \in \mathbb{R}^{n \times n}} \operatorname{tr}(H P)+\frac{1}{\eta}\|P\|_{1}, \\
\text { s.t. } \operatorname{tr} P=N, P=P^{\mathrm{T}}, 0 \leq P \leq I,
\end{gathered}
$$

where $\|\cdot\|_{1}$ is the entrywise $\ell_{1}$ matrix norm and $\eta$ is a penalty parameter for entrywise sparsity. The variational principle provides $P_{\infty, \eta}$ as a sparse representation of the projection operator onto the low-lying eigenspace. Numerical experiments in [16] demonstrate the localization of $P_{\infty, \eta}$ for the above $\ell_{1}$ regularized model.

For the finite temperature, applying the same $\ell_{1}$ regularization to enhance sparsity, we arrive at

$$
\begin{aligned}
& \min _{P \in \mathbb{R}^{n \times n}} \mathscr{E}_{\beta, \eta}(P)=\min _{P \in \mathbb{R}^{n \times n}} \operatorname{tr}(H P)+\frac{1}{\beta} \operatorname{tr}\{P \ln P+(1-P) \ln (1-P)\}+\frac{1}{\eta}\|P\|_{1} \\
& \text { s.t. } \quad \operatorname{tr} P=N, P=P^{\mathrm{T}}, 0 \leq P \leq I .
\end{aligned}
$$

Since this variational principle is strictly convex, the minimizer exists and is unique.

We will call the minimizing density matrix obtained by the above variational principles the localized density matrix (LDM). We provide in the remaining of this section some approximation results of LDM as the parameter $\eta \rightarrow \infty$. These results indicate 
that the proposed LDM can linearly approximate the originial density matrix with respect to the regularization parameter $\frac{1}{\eta}$ in both cases.

Theorem 1 (Consistency of LDM at zero temperature). Assume $H$ is non-degenerate that $\lambda_{N}<\lambda_{N+1}$ and denote $P_{\infty, \infty}$ the minimizer of [2]. Let $P_{\infty, \eta}$ be a minimizer of [11, we have

(a) $0 \leq \mathscr{E}_{\infty, \infty}\left(P_{\infty, \eta}\right)-\mathscr{E}_{\infty, \infty}\left(P_{\infty, \infty}\right) \leq \frac{1}{\eta}\left\|P_{\infty, \infty}\right\|_{1}$.

(b) $\left\|P_{\infty, \eta}-P_{\infty, \infty}\right\|_{F}^{2} \leq \frac{2}{\eta} \frac{\left\|P_{\infty, \infty}\right\|_{1}}{\left(\lambda_{N+1}-\lambda_{N}\right)}$.

In particular, $\lim _{\eta \rightarrow \infty} \mathscr{E}_{\infty, \infty}\left(P_{\infty, \eta}\right)=\mathscr{E}_{\infty, \infty}\left(P_{\infty, \infty}\right)$, and $\lim _{\eta \rightarrow \infty}\left\|P_{\infty, \eta}-P_{\infty, \infty}\right\|_{F}=0$.

Remark. Recall that the minimizer of [11] might not be unique [16], nevertheless the theorem applies to any minimizers.

Proof. It is clear that $\mathscr{E}_{\infty, \infty}\left(P_{\infty, \infty}\right) \leq \mathscr{E}_{\infty, \infty}\left(P_{\infty, \eta}\right)$ as $P_{\infty, \infty}$ minimizes the energy $\mathscr{E}_{\infty, \infty}$. On the other hand, since $P_{\eta}$ is an optimizer of [11], we have,

$$
\mathscr{E}_{\infty, \infty}\left(P_{\infty, \eta}\right) \leq \mathscr{E}_{\infty, \infty}\left(P_{\infty, \eta}\right)+\frac{1}{\eta}\left\|P_{\infty, \eta}\right\|_{1} \leq \mathscr{E}_{\infty, \infty}\left(P_{\infty, \infty}\right)+\frac{1}{\eta}\left\|P_{\infty, \infty}\right\|_{1}
$$

which yields the statement (a).

To show (b), we denote $\left\{\lambda_{i}, \phi_{i}\right\}_{i=1}^{n}$ the eigenpairs of $H$ and recall that

$$
P_{\infty, \infty}=\sum_{1 \leq i \leq N} \phi_{i} \phi_{i}^{\mathrm{T}}
$$

Moreover, as $P_{\infty, \eta}$ is symmetric and hence diagonalizable, we denote $\left\{\sigma_{i}, v_{i}\right\}_{i=1}^{n}$ its eigenpairs, with $P_{\infty, \eta} v_{i}=\sigma_{i} v_{i}$. Note that $\sum_{i} \sigma_{i}=N$ and $\sigma_{i} \in[0,1]$ since $P_{\infty, \eta}$ satisfies the constraint of 11.

Using the property of trace, we calculate

$$
\operatorname{tr}\left(H P_{\infty, \eta}\right)=\sum_{i=1}^{n}\left\langle\phi_{i}, H P_{\infty, \eta} \phi_{i}\right\rangle=\sum_{i=1}^{n} \lambda_{i}\left\langle\phi_{i}, P_{\infty, \eta} \phi_{i}\right\rangle=: \sum_{i=1}^{n} \lambda_{i} s_{i},
$$

where the last equality defines the shorthand notation $s_{i}=\left\langle\phi_{i}, P_{\infty, \eta} \phi_{i}\right\rangle$. Since $0 \preceq$ $P_{\infty, \eta} \leq I$ and $\operatorname{tr} P_{\infty, \eta}=N$, we have

$$
0 \leq s_{i} \leq 1, \quad \text { and } \quad \sum_{i=1}^{n} s_{i}=\sum_{i=1}^{n}\left\langle\phi_{i}, P_{\infty, \eta} \phi_{i}\right\rangle=\operatorname{tr} P_{\infty, \eta}=N .
$$

We now estimate, based on these properties of $\left\{s_{i}\right\}$,

$$
\begin{aligned}
& \frac{1}{\eta}\left\|P_{\infty, \infty}\right\|_{1} \geq \mathscr{E}_{\infty, \infty}\left(P_{\infty, \eta}\right)-\mathscr{E}_{\infty, \infty}\left(P_{\infty, \infty}\right) \\
&=\sum_{j=1}^{n} \lambda_{j} s_{j}-\sum_{j=1}^{N} \lambda_{j} \geq \sum_{j=1}^{N} \lambda_{j}\left(s_{j}-1\right)+\sum_{j=N+1}^{n} \lambda_{j} s_{j} \\
& \quad \geq \lambda_{N} \sum_{j=1}^{N}\left(s_{j}-1\right)+\lambda_{N+1} \sum_{j=N+1}^{n} s_{j}=\left(\lambda_{N+1}-\lambda_{N}\right) \sum_{j=1}^{N}\left(1-s_{j}\right) .
\end{aligned}
$$


This yields

$$
N-\sum_{j=1}^{N} s_{j}=\sum_{j=1}^{N}\left(1-s_{j}\right) \leq \frac{1}{\eta} \frac{\left\|P_{\infty, \infty}\right\|_{1}}{\left(\lambda_{N+1}-\lambda_{N}\right)} .
$$

We complete the proof of (b) by

$$
\begin{aligned}
\left\|P_{\infty, \eta}-P_{\infty, \infty}\right\|_{F}^{2} & =\operatorname{tr}\left(\left(P_{\infty, \eta}-P_{\infty, \infty}\right)^{2}\right) \\
& =\operatorname{tr}\left(P_{\infty, \eta}^{2}\right)-2 \operatorname{tr}\left(P_{\infty, \infty} P_{\infty, \eta}\right)+\operatorname{tr}\left(P_{\infty, \infty}^{2}\right) \\
& \leq \operatorname{tr}\left(P_{\infty, \eta}\right)-2 \operatorname{tr}\left(P_{\infty, \infty} P_{\infty, \eta}\right)+\operatorname{tr}\left(P_{\infty, \infty}\right) \\
& =2 N-2 \sum_{i=1}^{n}\left\langle\phi_{i}, P_{\infty, \infty} P_{\infty, \eta} \phi_{i}\right\rangle \\
& =2\left(N-\sum_{j=1}^{N} s_{j}\right) \leq \frac{2}{\eta} \frac{\left\|P_{\infty, \infty}\right\|_{1}}{\left(\lambda_{N+1}-\lambda_{N}\right)} .
\end{aligned}
$$

For the finite temperature case, we have the following analogous result.

Theorem 2 (Consistency of LDM at finite temperature). Denote $P_{\beta, \infty}$ and $P_{\beta, \eta}$ the minimizer of 4 and (12), respectively. It holds

$$
0 \leq \mathscr{E}_{\beta, \infty}\left(P_{\beta, \eta}\right)-\mathscr{E}_{\beta, \infty}\left(P_{\beta, \infty}\right) \leq \frac{1}{\eta}\left\|P_{\beta, \infty}\right\|_{1},
$$

and also the estimate

$$
\operatorname{tr}\left(\max \left(\beta^{-1},|H-\mu|\right)\left(P_{\beta, \eta}-P_{\beta, \infty}\right)^{2}\right) \leq \frac{1}{\eta}\left\|P_{\beta, \infty}\right\|_{1} .
$$

Remark. Note that as immediate consequence of 15, we have the estimate in Frobenius norm

$$
\left\|P_{\beta, \eta}-P_{\beta, \infty}\right\|_{F}^{2} \leq \frac{1}{\eta}\left\|P_{\beta, \infty}\right\|_{1} \min \left(\beta, \max _{i}\left|\lambda_{i}-\mu\right|^{-1}\right) .
$$

Taking the limit $\beta \rightarrow \infty$, as the chemical potential $\mu \rightarrow \frac{1}{2}\left(\lambda_{N}+\lambda_{N+1}\right)$, we get

$$
\lim _{\beta \rightarrow \infty} \min _{i}\left|\lambda_{i}-\mu\right| \rightarrow \frac{\lambda_{N+1}-\lambda_{N}}{2} .
$$

Therefore, we recover the estimate for zero temperature case (assuming $\lambda_{N}<\lambda_{N+1}$ ).

Proof. By optimality of $P_{\beta, \eta}$ and $P_{\beta, \infty}$, we have $\mathscr{E}_{\beta, \infty}\left(P_{\beta, \infty}\right) \leq \mathscr{E}_{\beta, \infty}\left(P_{\beta, \eta}\right)$ and

$$
\text { (17) } \begin{aligned}
\frac{1}{\eta}\left\|P_{\beta, \infty}\right\|_{1} \geq \mathscr{E}_{\beta, \infty}\left(P_{\beta, \eta}\right)-\mathscr{E}_{\beta, \infty}\left(P_{\beta, \infty}\right) & \\
& =\operatorname{tr}\left(\left(P_{\beta, \eta}-P_{\beta, \infty}\right) H\right)+\beta^{-1} \operatorname{tr}\left(\varphi\left(P_{\beta, \eta}\right)-\varphi\left(P_{\beta, \infty}\right)\right) .
\end{aligned}
$$

Hence, we obtain the first conclusion (14) of the theorem.

Recall that $\varphi^{\prime}(x)=\ln x-\ln (1-x)$ and hence by explicit calculation using 9

$$
\varphi^{\prime}\left(P_{\beta, \infty}\right)=\ln \left(P_{\beta, \infty}\left(I-P_{\beta, \infty}\right)^{-1}\right)=-\beta(H-\mu) .
$$


Therefore

$$
\operatorname{tr}\left(\left(P_{\beta, \eta}-P_{\beta, \infty}\right) H\right)=\operatorname{tr}\left(\left(P_{\beta, \eta}-P_{\beta, \infty}\right)(H-\mu)\right)=-\beta^{-1} \operatorname{tr}\left(\varphi^{\prime}\left(P_{\beta, \infty}\right)\left(P_{\beta, \eta}-P_{\beta, \infty}\right)\right),
$$

where we have used that $\operatorname{tr} P_{\beta, \eta}=\operatorname{tr} P_{\beta, \infty}=N$ in the first equality. Substitute into (17), we get

$$
\frac{1}{\eta}\left\|P_{\beta, \infty}\right\|_{1} \geq \beta^{-1}\left[\operatorname{tr}\left(\varphi\left(P_{\beta, \eta}\right)-\varphi\left(P_{\beta, \infty}\right)\right)-\operatorname{tr}\left(\varphi^{\prime}\left(P_{\beta, \infty}\right)\left(P_{\beta, \eta}-P_{\beta, \infty}\right)\right)\right]
$$

Note that the right hand side is the Bregman divergence of $\varphi$. For $x, y \in[0,1]$, we have

$$
\varphi(x)-\varphi(y)-\varphi^{\prime}(y)(x-y)=x(\ln x-\ln y)+(1-x)(\ln (1-x)-\ln (1-y)),
$$

which is the Fermi-Dirac relative entropy. Following a similar calculation in 14 . Theorem 1] (see also an improved version in [9]), we minimize $x \in[0,1]$ for fixed $y$ and find

$$
x \ln \frac{x}{y}+(1-x) \ln \frac{1-x}{1-y} \geq \frac{\ln \frac{1-y}{y}}{1-2 y}(x-y)^{2} .
$$

Explicit calculation verifies that for $y \in[0,1]$, we have

$$
\frac{\ln \frac{1-y}{y}}{1-2 y} \geq \max \left(\left|\ln \frac{1-y}{y}\right|, 1\right) .
$$

Hence, combining with [18 and using Klein's lemma [29. Theorem 2.5.2], we arrived at the estimate 15

$$
\frac{1}{\eta}\left\|P_{\beta, \infty}\right\|_{1} \geq \operatorname{tr}\left(\max \left(\beta^{-1},|H-\mu|\right)\left(P_{\beta, \eta}-P_{\beta, \infty}\right)^{2}\right) .
$$

\section{NUMERICAL ALGORITHMS FOR LDMS}

3.1. Computing LDM at zero temperature. In $[16$, a numerical algorithm has been proposed to solve 11 based on Bregman iteration. Bregman iteration was first introduced into information science for solving total variation related problems as an analog of "adding back the noise" in image denoising [25]. Split Bregman iteration has been later proposed in [13] based on the idea of variable splitting. These algorithms have since received intensive attention due to its efficiency in many $\ell_{1}$ related constrained optimization problems [34 35]. The equivalence of the Bregman iteration with the alternating direction method of multipliers (ADMM), Douglas-Rachford splitting and aug-

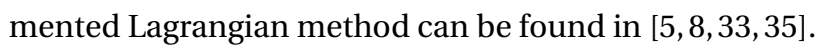

Let's first recall the algorithm proposed in $[16$. By introducing auxiliary variables $Q$ and $R$, the optimization problem [11] is equivalent to

$$
\begin{aligned}
& \min _{P, Q, R \in \mathbb{R}^{n \times n}} \frac{1}{\eta}\|Q\|_{1}+\operatorname{tr}(H P) \\
& \text { s.t. } Q=P, R=P, \operatorname{tr} P=N, 0 \leq R \leq I,
\end{aligned}
$$


The method of Bregman iteration suggests to approach [19] by solving:

$$
\begin{aligned}
& \left(P^{k}, Q^{k}, R^{k}\right)=\arg \min _{P, Q, R \in \mathbb{R}^{n \times n}} \frac{1}{\eta}\|Q\|_{1}+\operatorname{tr}(H P) \\
& +\frac{\lambda}{2}\left\|P-Q+B^{k-1}\right\|_{F}^{2}+\frac{r}{2}\left\|P-R+D^{k-1}\right\|_{F}^{2} \\
& \text { s.t. } \quad \operatorname{tr} P=N, 0 \leq R \leq I, \\
& B^{k}=B^{k-1}+P^{k}-Q^{k} \text {, } \\
& D^{k}=D^{k-1}+P^{k}-R^{k} \text {, }
\end{aligned}
$$

where variables $B, D$ are essentially Lagrangian multipliers and parameters $r, \lambda$ control the penalty terms. Solving $P^{k}, Q^{k}, R^{k}$ in $[20$ alternatively leads to algorithm 1 proposed in [16].

$$
\begin{aligned}
& 1 \text { Initialize } Q^{0}=R^{0}=P^{0} \in \mathscr{C}=\left\{P \in \mathbb{R}^{n \times n} \mid P=P^{\mathrm{T}}, \operatorname{tr} P=N, 0 \leq P \leq I\right\}, B^{0}=D^{0}=0 \\
& 2 \text { while not converged do } \\
& 3 \quad P^{k}=\Gamma^{k}-\frac{\operatorname{tr}\left(\Gamma^{k}\right)-N}{n} \text {, where } \Gamma^{k}= \\
& \frac{\lambda}{\lambda+r}\left(Q^{k-1}-B^{k-1}\right)+\frac{r}{\lambda+r}\left(R^{k-1}-D^{k-1}\right)-\frac{1}{\lambda+r} H . \\
& Q^{k}=\operatorname{Shrink}\left(P^{k}+B^{k-1}, \frac{1}{\lambda \eta}\right)=\operatorname{sign}\left(P^{k}+B^{k-1}\right) \max \left\{\left|P^{k}+B^{k-1}\right|-\frac{1}{\lambda \eta}, 0\right\} \text {. } \\
& R^{k}=V \min \{\max \{\Lambda, 0\}, 1\} V^{T} \text {, where }[V, \Lambda]=\operatorname{eig}\left(P^{k}+D^{k-1}\right) . \\
& B^{k}=B^{k-1}+P^{k}-Q^{k} \text {. } \\
& D^{k}=D^{k-1}+P^{k}-R^{k} \text {. }
\end{aligned}
$$

Algorithm 1: Zero temperature localized density matrix minimization

3.2. Computing LDM at finite temperature. Similarly, the LDM for the finite temperature case proposed in (12) can also be solved based on Bregman iteration. By introducing auxiliary variables $Q$ and $R$, the optimization problem $[12$ is equivalent to

$$
\begin{aligned}
& \min _{P, Q, R \in \mathbb{R}^{n \times n}} \frac{1}{\eta}\|Q\|_{1}+\operatorname{tr}(H P)+\frac{1}{\beta} \operatorname{tr}\{P \ln P+(1-P) \ln (1-P)\} \\
& \text { s.t. } Q=P, R=P, \operatorname{tr} Q=N, 0 \leq R \leq I .
\end{aligned}
$$


Note that we have explored the flexibility of the augmenting approach to impose the trace constraint on $Q$, which is equivalent of imposing the constraint on $P$. The minimization (19) can be iteratively solved by:

$$
\begin{aligned}
&\left(P^{k}, Q^{k}, R^{k}\right)= \arg \min _{P, Q, R \in \mathbb{R}^{n \times n}} \frac{1}{\eta}\|Q\|_{1}+\operatorname{tr}(H P)+\frac{1}{\beta} \operatorname{tr}\{P \ln P+(1-P) \ln (1-P)\} \\
&+\frac{\lambda}{2}\left\|P-Q+B^{k-1}\right\|_{F}^{2}+\frac{r}{2}\left\|P-R+D^{k-1}\right\|_{F}^{2} \\
& \text { s.t. } \quad \operatorname{tr} Q=N, 0 \leq R \leq I, \\
& B^{k}=B^{k-1}+P^{k}-Q^{k}, \\
& D^{k}=D^{k-1}+P^{k}-R^{k},
\end{aligned}
$$

where variables $B, D$ and parameters $r, \lambda$ have the similar roles as in 20. Solving $P^{k}, Q^{k}, R^{k}$ in (24) alternatively leads to the following three sub-optimization problems.

$$
\begin{aligned}
& 1^{\circ} P^{k}=\arg \min _{P \in \mathbb{R}^{n \times n}} \operatorname{tr}(H P)+\frac{1}{\beta} \operatorname{tr}\{P \ln P+(1-P) \ln (1-P)\}+\frac{\lambda}{2}\left\|P-Q^{k-1}+B^{k-1}\right\|_{F}^{2} \\
& +\frac{r}{2}\left\|P-R^{k-1}+D^{k-1}\right\|_{F}^{2} . \\
& 2^{\circ} Q^{k}=\arg \min _{Q \in \mathbb{R}^{n \times n}} \frac{1}{\eta}\|Q\|_{1}+\frac{\lambda}{2}\left\|P^{k}-Q+B^{k-1}\right\|_{F}^{2}, \quad \text { s.t. } \quad \operatorname{tr} Q=N . \\
& 3^{\circ} R^{k}=\arg \min _{R \in \mathbb{R}^{n \times n}}\left\|P^{k}-R+D^{k-1}\right\|_{F}^{2}, \quad \text { s.t. } \quad 0 \leq R \leq I .
\end{aligned}
$$

Note that the sub-minimization problem $3^{\circ}$ of algorithm 2 can be solved explicitly, similarly as before

$$
R^{k}=V \min \{\max \{\Lambda, 0\}, 1\} V^{T}, \text { where }[V, \Lambda]=\operatorname{eig}\left(P^{k}+D^{k-1}\right) .
$$

To solve the sub-problem $2^{\circ}$ of algorithm 2 let's denote by $Q_{o}$ the off-diagonal part of $Q$ and write $Q_{d}$ as the diagonal vector of $Q$. Namely, we have $Q=Q_{o}+\operatorname{diag}\left(Q_{d}\right)$. We also use similar notations for all other matrices. Then the solution of the sub-problem $2^{\circ}$ can be written as $Q^{k}=Q_{o}^{k}+\operatorname{diag}\left(Q_{d}^{k}\right)$, where $Q_{o}^{k}$ and $Q_{d}^{k}$ are given by (denoting $M^{k}=$ $\left.P^{k}+B^{k-1}\right)$

$$
\begin{aligned}
& Q_{o}^{k}=\operatorname{Shrink}\left(M_{o}^{k},(\lambda \eta)^{-1}\right)=\operatorname{sign}\left(M_{o}^{k}\right) \max \left\{\left|M_{o}^{k}\right|-(\lambda \eta)^{-1}, 0\right\}, \\
& Q_{d}^{k}=\arg \min _{Q_{d} \in \mathbb{R}^{n}} \frac{1}{\eta}\left\|Q_{d}\right\|_{1}+\frac{\lambda}{2}\left\|Q_{d}-M_{d}^{k}\right\|^{2}, \quad \text { s.t. } \quad \mathbf{1}^{T} Q_{d}=N .
\end{aligned}
$$

here, 1 is a $n \times 1$ vector with all elements 1 . Note that 26 is a convex optimization problem with size $n$, which can be efficiently solved using the following Bregman iteration.

$$
\begin{aligned}
Q_{d}^{k, s} & =\operatorname{Shrink}\left(\frac{\lambda}{\lambda+r} M_{d}^{k}+\frac{r}{\lambda+r}\left(v^{s-1}-b^{s-1}\right), \frac{1}{(\lambda+r) \eta}\right) . \\
v^{s} & =Q_{d}^{k, s}+b^{s-1}-\frac{1}{n}\left(\mathbf{1}^{T}\left(Q_{d}^{k, s}+b^{s-1}\right)-N\right) . \\
b^{s} & =b^{s-1}+Q_{d}^{k, s}-v^{s} .
\end{aligned}
$$


Next, we propose to iteratively solve the sub-minimization $1^{\circ}$ in algorithm 2 .

$$
\begin{aligned}
P^{k}=\arg \min _{P \in \mathbb{R}^{n \times n}} \operatorname{tr}(H P)+\frac{1}{\beta} \operatorname{tr}\{P \ln P+(1-P) \ln (1-P)\} & +\frac{\lambda}{2}\left\|P-Q^{k-1}+B^{k-1}\right\|_{F}^{2} \\
& +\frac{r}{2}\left\|P-R^{k-1}+D^{k-1}\right\|_{F}^{2} .
\end{aligned}
$$

Note that this is a convex problem, and hence the existence and uniqueness of $P^{k}$ are guaranteed. By the KKT condition, $P^{k}$ satisfies

$$
H+\frac{1}{\beta}\left(\ln P^{k}-\ln \left(1-P^{k}\right)\right)+\lambda\left(P^{k}-Q^{k-1}+B^{k-1}\right)+r\left(P^{k}-R^{k-1}+D^{k-1}\right)=0 .
$$

Equivalently, we may write the above equation as

$$
P^{k}=\left[1+\exp \left(\beta\left(H+\lambda\left(P^{k}-Q^{k-1}+B^{k-1}\right)+r\left(P^{k}-R^{k-1}+D^{k-1}\right)\right)\right)\right]^{-1} .
$$

Thus a natural way to solve for $P^{k}$ is to use a fix point iteration given by

$$
\begin{aligned}
& Z^{l+1}=\frac{1}{1+\exp \left(\beta Y^{l}\right)} ; \\
& Y^{l}=H+\lambda\left(Z^{l}-Q^{k-1}+B^{k-1}\right)+r\left(Z^{l}-R^{k-1}+D^{k-1}\right) .
\end{aligned}
$$

The following proposition gives the convergence of the above scheme.

Proposition 3. Assume $\beta(\lambda+r)<4$. Given any symmetric matrices $Q, R, B, D \in \mathbb{R}^{n \times n}$, the iteration scheme

$$
\begin{aligned}
& Z^{l+1}=\frac{1}{1+\exp \left(\beta Y^{l}\right)} ; \\
& Y^{l}=H+\lambda\left(Z^{l}-Q+B\right)+r\left(Z^{l}-R+D\right)
\end{aligned}
$$

converges exponentially as $l \rightarrow \infty$ :

$$
\left\|Z^{l}-Z^{*}\right\|=(\beta(\lambda+r) / 4)^{l}\left\|Z^{0}-Z^{*}\right\|,
$$

where $Z^{*}$ is the unique solution of 28 .

Proof. Denote the Fermi-Dirac function

$$
\phi_{\beta}(x)=\frac{1}{1+\exp (\beta x)} .
$$

We have then

$$
\max _{x}\left|\phi_{\beta}^{\prime}(x)\right|=\max _{x}\left|\frac{\beta \exp (\beta x)}{(1+\exp (\beta x))^{2}}\right| \leq \beta / 4 .
$$

Therefore,

$$
\left\|Z^{l+1}-Z^{*}\right\|=\left\|\phi_{\beta}\left(Y^{l}\right)-\phi_{\beta}\left(Y^{*}\right)\right\| \leq \frac{\beta}{4}\left\|Y^{l}-Y^{*}\right\|=\frac{\beta(\lambda+r)}{4}\left\|Z^{l}-Z^{*}\right\|,
$$

where $Y^{*}=H+\lambda\left(Z^{*}-Q+B\right)+r\left(Z^{*}-R+D\right)$. The proposition then follows by iterating with respect to $l$.

In summary, we arrive at algorithm 2 for (12). 


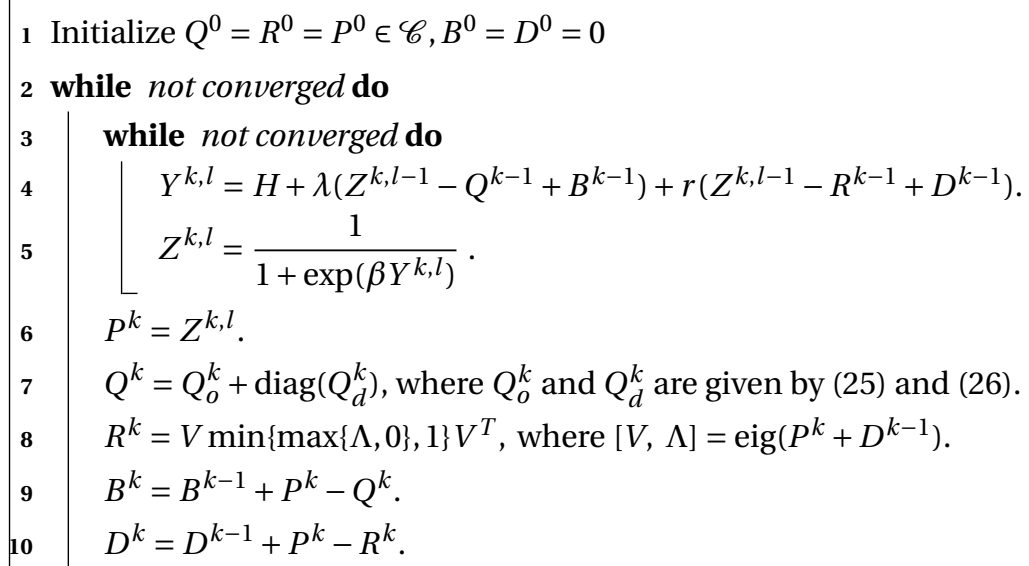

Algorithm 2: Finite temperature localized density matrix minimization.

Remark. In practice, it is not necessary to require all inner iterations' convergence in algorithm 2 In our numerical experiments, we run all the inner iterations in algorithm 2 for a given small number of steps, as $Z^{l}$ converges exponentially by Proposition 3

Theorem 4 (Convergence of Algorithm 1 and Algorithm 2).

(1) The sequence $\left\{\left(P^{k}, Q^{k}, R^{k}\right)\right\}_{k}$ generated by algorithm 1 from any starting point converges to a minimum of the variational problem (11).

(2) The sequence $\left\{\left(P^{k}, Q^{k}, R^{k}\right)\right\}_{k}$ generated by algorithm 2 from any starting point converges to a minimum of the variational problem 12 .

Proof. The convergence of algorithm 1 is proved in [16]. The proof in fact also applies verbatim to algorithm 2 as it is written for generic convex energy $E(P)$.

While the minimization problems [11 and [12) are convex and the proposed algorithms converge to the minimizers by theorem 4 it is also clear that the computational efficiency of the algorithms 1 and 2 is limited by the eigen-decomposition in the step of eigenvalue thresholding, and also the inner iteration in the finite temperature case. The computational cost for standard eigen-decompsition algorithm is $\mathscr{O}\left(n^{3}\right)$, which is rather expensive for large scale calculations. In the next section, inspired by ideas from linear-scaling algorithms for electronic structure, we propose approximate algorithms to solve [11] and (12) by replacing eigendecompsition with polynomial functions acting on matrices. The resulting algorithms have computational cost linearly scaling with the matrix size $n$.

\section{APPROXIMATION BY BANDED MATRICES AND LINEAR-SCALING ALGORITHMS}

4.1. Banded matrices. Based on results indicated in theorems 1 and 2 the proposed LDM serves as a nice approximation of the true density matrix. More importantly, similar to many $\ell_{1}$ regularization methods developed for compressed sensing problems, 
the minimizing density matrices are expected to have certain sparse structure. More precisely, we denote the set of all banded matrices with band width $w$ as

$$
\mathscr{B}_{w}=\left\{P=\left(p_{i j}\right) \in \mathbb{R}^{n \times n} \mid P=P^{T}, p_{i j}=0, \forall j \notin \mathscr{N}_{i}^{w}\right\},
$$

where $\mathscr{N}_{i}^{w}$ denotes a $w$-neighborhood of $i$. In particular, for the $1 D$ examples with the periodic boundary condition considered later in this paper, the neighborhood is chosen as

$$
\mathscr{N}_{i}^{w}=\{j \in\{1,2, \ldots, n\}|| i-j \mid \bmod n \leq w\},
$$

for a given band width $w \in\left\{0,1,2, \cdots,\left[\frac{n}{2}\right]\right\}$ ( $w$ is typically chosen much smaller than $n / 2$ ). For example, one can consider a banded discretized Hamiltonian $H$ with the periodic boundary condition (e.g., a central difference discretization of Hamiltonian $\left.-\frac{1}{2} \Delta+V\right)$. Numerical results in 16 suggest that the LDM is banded with a small band width. We remark that, however, theoretical validation of this observation is still open and remains to be investigated in future works.

This motivates the following variational problems to approximate the LDMs proposed in (11) and (12) by simply constraining the problems on the set of banded matrices.

$$
\begin{gathered}
\min _{P \in \mathbb{R}^{n \times n}} \mathscr{E}_{\infty, \eta}^{w}(P)=\min _{P \in \mathbb{R}^{n \times n}} \operatorname{tr}(H P)+\frac{1}{\eta}\|P\|_{1}, \\
\text { s.t. } \operatorname{tr} P=N, P=P^{\mathrm{T}}, 0 \leq P \leq I, P \in \mathscr{B}_{w} . \\
\min _{P \in \mathbb{R}^{n \times n}} \mathscr{E}_{\beta, \eta}^{w}(P)=\min _{P \in \mathbb{R}^{n \times n}} \operatorname{tr}(H P)+\frac{1}{\beta} \operatorname{tr}\{P \ln P+(1-P) \ln (1-P)\}+\frac{1}{\eta}\|P\|_{1} \\
\text { s.t. } \operatorname{tr} P=N, P=P^{\mathrm{T}}, 0 \leq P \leq I, P \in \mathscr{B}_{w} .
\end{gathered}
$$

Note that the above two optimization problems are still convex. This is in contrast to the usual minimization problems developed in the literature of linear-scaling algorithms [4.21.

4.2. A linear-scaling algorithm for the zero temperature case. The advantage of considering banded matrices is that it allows for linear-scaling algorithms. Let us consider first the eigenvalue thresholding:

$$
R=V \min \{\max \{\Lambda, 0\}, 1\} V^{T}, \text { where }[V, \Lambda]=\operatorname{eig}(M) .
$$

Observe that the above formula can be written as a matrix function

$$
R=h(M), \quad \text { where } h(x)=\min \{\max \{x, 0\}, 1\}=\min \{1 / 2(|x|+x), 1\} .
$$

The standard evaluation of the matrix function $h$ using spectral theory needs diagonalization as in 37). The key idea is to approximate general matrix functions by polynomials, as the polynomials acting on matrix only involves products and sums, which can take advantage of the bandedness of the matrix. This type of algorithms has been explored extensively in the literature of linear scaling algorithms (see [12] and the review

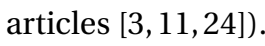


Specifically, in the current context, we will approximate the hard thresholding function $h(x)=\min \{1 / 2(|x|+x), 1\}$ using the Chebyshev polynomial approximation [31]. Recall that the standard Chebyshev polynomials approximation minimizes the $L^{\infty}$ error on the interval $[-1,1]$. Hence, we will first rescale the matrix such that its eigenvalues lie in the interval. The Chebyshev polynomial approximation here essentially leads to approximate the matrix in term of minimizing the matrix norm error.

For this, we first use the power method to estimate the largest eigenvalue $\lambda_{\max }$ and the smallest eigenvalue $\lambda_{\min }$ of $M$. An affine scaling of $M$ gives $s(M)$, whose eigenvalue is in $[-1,1]$, where

$$
s(M)=\frac{2}{\lambda_{\max }-\lambda_{\min }}\left(M-\lambda_{\min }\right)-1 .
$$

Note that its inverse is given by

$$
s^{-1}(M)=\frac{\lambda_{\max }-\lambda_{\min }}{2} M+\frac{\lambda_{\min }+\lambda_{\max }}{2} .
$$

We approximate

$$
h(M)=h \circ s^{-1}(s(M)) \approx T_{H T}^{m}(s(M)),
$$

where $T_{H T}^{m}$ is the Chebyshev polynomial approximation of $h \circ s^{-1}$ on $[-1,1]$. Figure 1 (a1, a2) illustrates the Chebyshev polynomial approximation of $h$ (i.e., $h \circ s^{-1}$ assuming the scaling function $s$ is identity) by 40 degree polynomials with approximation error within $10^{-2}$. Higher degree polynomial is needed if $M$ has a larger spectrum span. We point out that the approximation error can be further improved if a better polynomial approximation can be chosen, which will be investigated in our future work. In our numerical tests, it seems that fixing the degree $\leq 50$ gives satisfactory result for the test examples.

As acting $T_{H T}^{m}$ on $s(M)$ involves matrix products which will increase the matrix bandwidth. The resulting matrix is projected (with respect to Frobenius norm) on the space $\mathscr{B}_{w}$ to satisfy the constraint. This projection is explicitly given by the truncation operator $\mathfrak{T}_{w}: \mathbb{R}^{n \times n} \rightarrow \mathscr{B}_{w}$ which sets all matrix entries to 0 outside the $w$-band.

To summarize, the eigenvalue thresholding can be approximated by algorithm 3 . For

$1 \widehat{P}=$ EigenThresviaChebyPoly $(P, m)$

Input: $P \in \mathscr{B}_{w}, m$

Output: $\widehat{P} \in \mathscr{B}_{w}$

2 Estimate $\lambda_{\max }$ and $\lambda_{\min }$ of $P$ using the power method.

3 Compute $T_{H T}^{m}(x)$ as the Chebyshev polynomial approximation of $h\left(\frac{\lambda_{\max }-\lambda_{\min }}{2} x+\frac{\lambda_{\min }+\lambda_{\max }}{2}\right)$.

$4 P_{\text {new }}=\frac{2}{\lambda_{\max }-\lambda_{\min }}\left(P-\lambda_{\min }\right)-1$.

$5 \widehat{P}=\mathfrak{T}_{w} T_{H T}^{m}\left(P_{\text {new }}\right)$.

Algorithm 3: A linear-scaling algorithm for eigenvalue thresholding.

banded matrix with band width $w$, the computational cost of this algorithm is $O\left(n m^{2} w\right)$ 
which scales linearly with respect to the matrix dimension $n$. Therefore, for matrices in $\mathscr{B}_{w}$, this algorithm has much better computational efficiency in particular for matrices of large size.

4.3. A linear-scaling algorithm for the finite temperature case. Replacing the eigenvalue thresholding in algorithm 1 by algorithm 3 we obtain the following algorithm for computing LDM in the zero temperature case. We remark that all the steps in al-

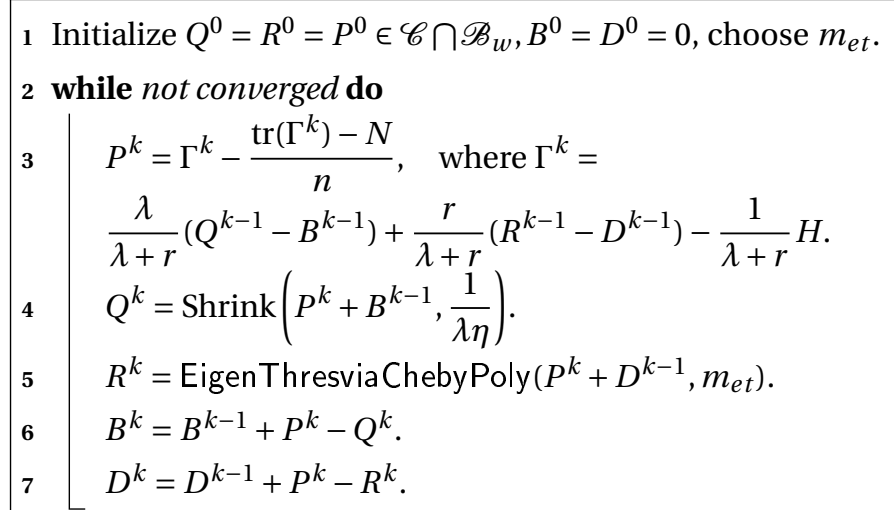

Algorithm 4: A linear-scaling algorithm for computing LDM at zero temperature.

gorithm 4 preserve the bandedness of the matrices: For the approximate eigenvalue thresholding, this is guaranteed by the explicit projection step; it can be easily checked for the other steps. Hence, the iterates of the algorithm $(Q, R, P, B, D)$ will remain in $\mathscr{B}_{w}$ as the initial condition lies in the set. Thus, the whole algorithm is linearly scaling.

For the finite temperature case, we need a further approximation for evaluating the Fermi-Dirac matrix function $(1+\exp (\beta Y))^{-1}$, as direct computation also involves matrix diagonalization, which is $\mathscr{O}\left(n^{3}\right)$. Following the same procedure as in algorithm 3 we can achieve a linear scaling algorithm by a Chebyshev polynomial approximation of the Fermi-Dirac function $\phi_{\beta}(x)=(1+\exp (\beta x))^{-1}$.

This leads to an algorithm FermiDiracviaChebyPoly $(Y, m)$ for approximating the FermiDirac operation by simply replacing the hard thresholding function $h(x)$ with the FermiDirac function $\phi_{\beta}(x)$ in algorithm 3 Hence, we omit the details. Figure 1 (b1, b2) shows the approximation of $\phi_{\beta}(x)$ for $\beta=10$ with a degree 40 polynomial. Nice agreement is observed. We remark that if the temperature is lower (so that $\beta$ is larger), a higher degree polynomial is needed as the function $\phi_{\beta}(x)$ has larger derivatives. In fact, as $\beta \rightarrow \infty, \phi_{\beta}$ converges to a Heaviside function with jump at $x=0$. Thus, we arrive at the following algorithm 5 for computing LDM of the finite temperature case with linear scale computation cost with respect to the matrix size $n$.

We remark that since polynomial products are applied to approximate the hard thresholding function and the Fermi-Dirac operation, approximation errors have been introduced in each iteration of the proposed algorithms 4 and 5 Therefore, the convergence 

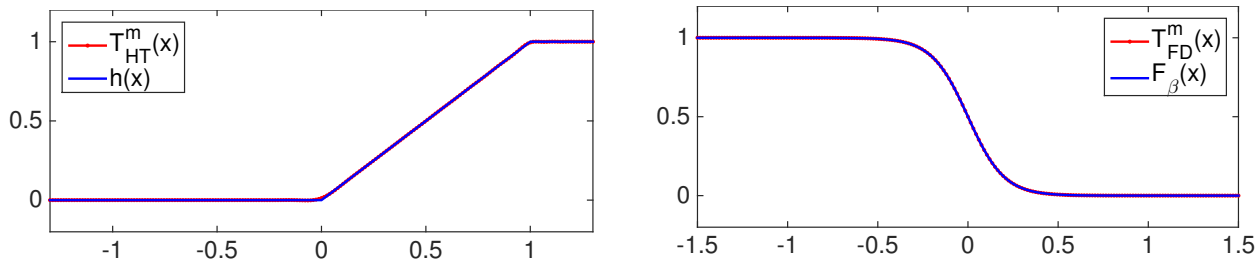

(a1)

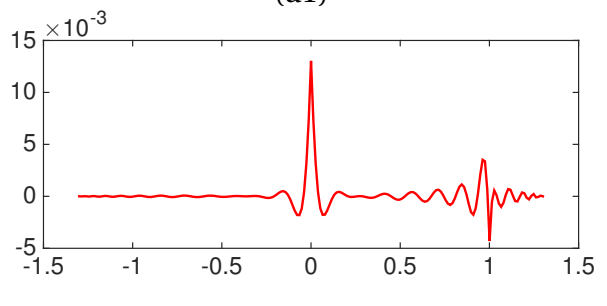

(b1)

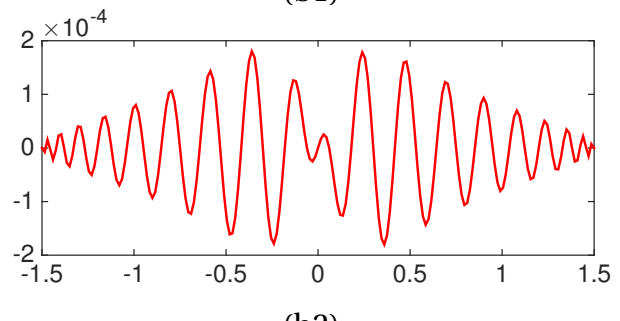

(b2)

FIGURE 1. (a1): Chebyshev approximation of $h(x)$ with $m=50$ degree polynomial. (a2): The difference between Chebyshev approximation and $h(x)$. (b1): Chebyshev approximation of $\phi_{\beta}(x)$ for $\beta=10$ with $m=40$ degree polynomial. (b2): The difference between Chebyshev approximation and $\phi_{\beta}(x)$.

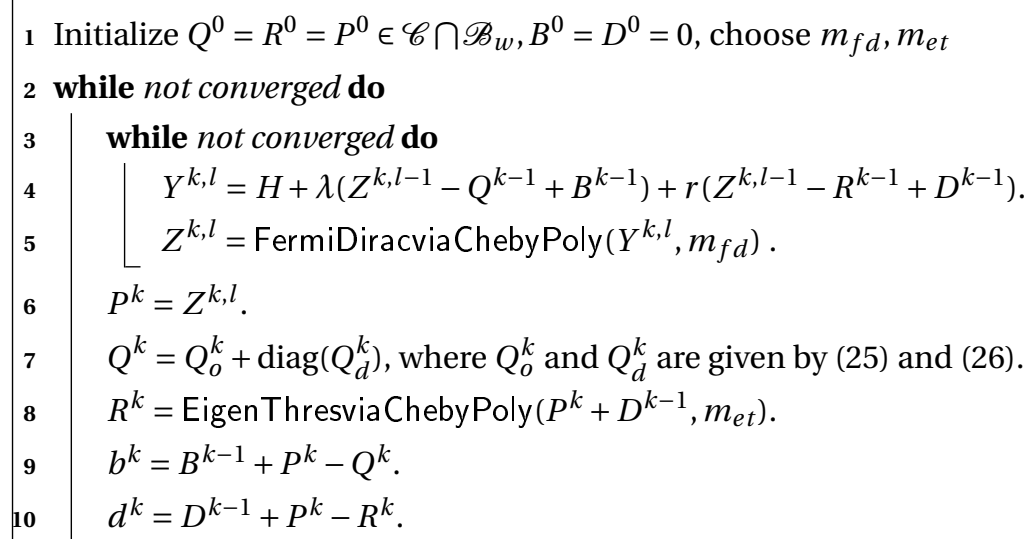

Algorithm 5: A linear-scaling algorithm for computing LDM at finite temperature.

proof used in theorem 4 as we discussed in 16 can not be directly applied, although our numerical results in Section 5 illustrate satisfactory approximation to model 2 and model 4 Note that similar issues arise in theoretical understanding of convergence of other iterative linear-scaling algorithms, for example 10. 


\section{NUMERICAL EXPERIMENTS}

In this section, numerical experiments are presented to demonstrate the proposed models and algorithms for LDM computing for at zero and finite temperatures. We conduct numerical comparisons of our results between LDM computation with and without the linear-scaling algorithms, which indicates satisfactory results of the proposed linear-scaling algorithms based on approximation by banded matrices. We further illustrate efficiency of the proposed linear scaling algorithms. All numerical experiments are implemented in MATLAB in a PC with a $16 \mathrm{~Gb}$ RAM and a $2.7 \mathrm{GHz}$ quad-core CPU.

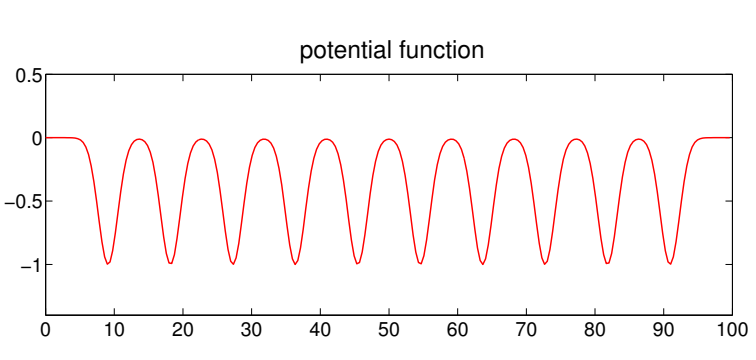

(a)

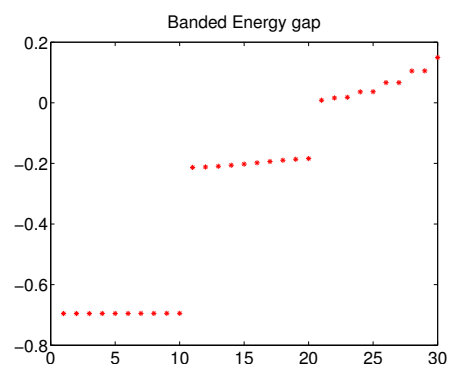

(b)

FIgURE 2. (a): The potential function in the GW model. (b): The spectrum of the (discretized) Hamiltonian operator.

In our experiments, we consider the proposed models defined on $1 \mathrm{D}$ domain $\Omega=$ $[0,100]$ with periodic boundary condition. In this case, the matrix $H$ is a discretization of the Schrödinger operator $-\frac{1}{2} \Delta+V$ defined on $\Omega$. Here, $\Delta$ is approximated by a central difference on $[0,100]$ with equally spaced 400 points. In addition, we consider a potential $V$ as a sum of Gaussian wells so that the potential is given by

$$
V(x)=-V_{0} \sum_{j=1}^{N_{\mathrm{at}}} \exp \left[-\frac{\left(x-x_{j}\right)^{2}}{\delta^{2}}\right],
$$

where $N_{\text {at }}$ gives the number of potential wells. We brief this potential as GW for convenience. In our numerical experiments, we choose $N_{\text {at }}=10$ and $x_{j}=100 j / 11$ for $j=1, \ldots, N_{\text {at }}$. The potential is plotted in Figure 2(a). For this given potential, the Hamiltonian operator $H=-\frac{1}{2} \Delta+V(x)$ exhibits two low-energy bands separated by finite gaps from the rest of the eigenvalue spectrum (See Figure $2(b)$ ).

The first experiment demonstrates the proposed methods at zero temperature for an insulating case, where we set parameters as $\eta=100, N=10, m_{e t}=50$. Figure 3(a) illustrates the true density matrix $\sum_{1 \leq i \leq 10} \phi_{i} \phi_{i}^{T}$ obtained by the first 10 eigenfunctions $\left\{\phi_{i}\right\}_{i=1}^{10}$ of $H$. Figure 3 (b) plots the density matrix obtained from the proposed model using algorithm 1 . As one can observe from Figure $3(\mathrm{~b})$, the LDM provides a quite good approximation to the true density matrix. Note that the LDM with a larger $\eta$ imposes a smaller penalty on the sparsity, and hence the solution has a wider spread and closer to 
the true density matrix. The approximation behavior is stated in theorem 1 . We also refer our previous work 16 for more numerical discussion about this point. Figure $3(\mathrm{c}, \mathrm{d})$ illustrate computing results of LDM using algorithm 4 with band size $w=10,20$ respectively. As we can see from the results, a moderate size of band width can provide satisfactory approximation for the LDM. In addition, we also conduct quantitative comparisons between algorithm 1 and algorithm 4 in table 1 where $P$ and $P_{w}$ are results obtained from algorithm 1 and algorithm 4 respectively. The relative energy error $\frac{\left|\operatorname{tr}(H P)-\operatorname{tr}\left(H P_{w}\right)\right|}{|\operatorname{tr}(H P)|}$ and the comparable relative differences between $\frac{\left\|P-P_{w}\right\|_{F}}{\|P\|_{F}}$ and $\frac{\left\|\mathfrak{T}_{w}(P)-P_{w}\right\|_{F}}{\|P\|_{F}}$ indicate that results obtained by linear-scaling algorithm 4 can provide good estimation of the LDM created from algorithm 1

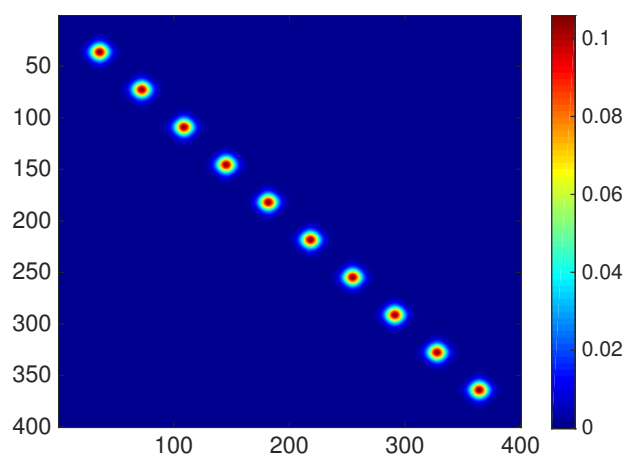

(a)

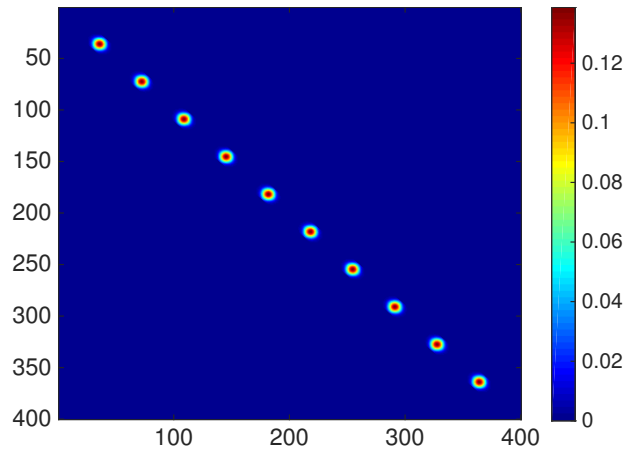

(c)

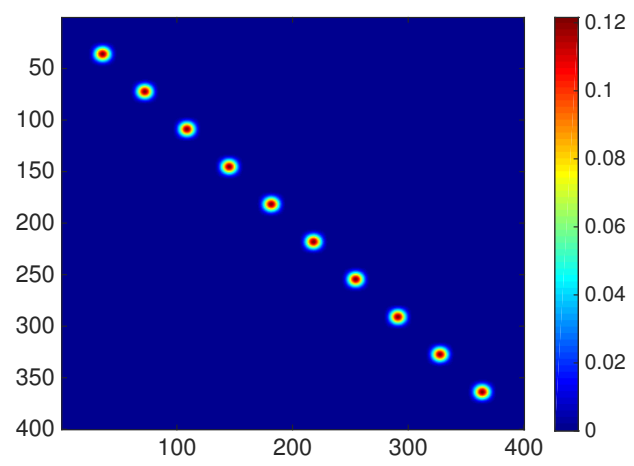

(b)

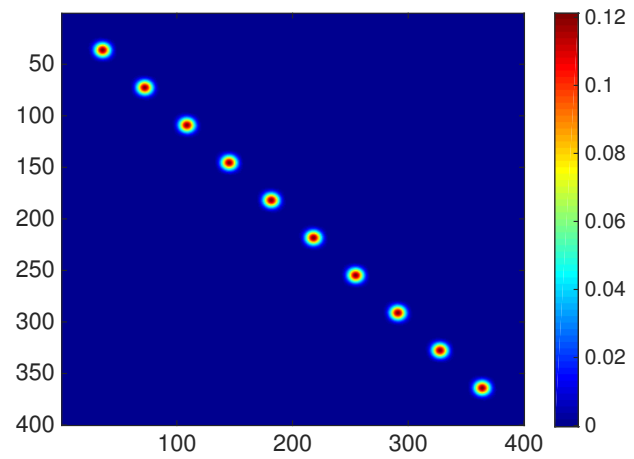

(d)

FIGURE 3. Result comparisons of the zero temperature case with GW potential ( $n=400, N=10$ ). (a). The true density function obtained by the first $\mathrm{N}$ eigenfunctions of $H$. (b). Solution of the localized density matrix with $\eta=100$ using algorithm 1 (c), (d). Solutions of the localized density matrices $(\eta=100)$ using algorithm 4 with $w=10,20$ respectively.

For the finite temperature case, it is known that the true density matrix decays exponentially fast along the off-diagonal direction. In the second experiment, we test the 
algorithms for the finite temperature model with potential free case and GW case. We set parameters $\eta=100, \beta=1, m_{e t}=40, m_{f d}=20$ for both cases. Figure 4 (a) and Figure 5 (a) illustrate the corresponding true density matrices described by $\sum_{i} \rho_{i} \phi_{i} \phi_{i}^{T}$ using (9), whose non-zero entries concentrate on a narrow band along the diagonal direction. Figure $4(\mathrm{~b})$ and Figure 5 (b) plot the density matrix obtained from the proposed model using algorithm 2 It is clear that the LDM performs reasonably good approximation to the true density matrix. Figure 4 (c, d) and Figure 5 (c, d) illustrate computing results of LDM using algorithm 5 with band size $w=10,20$ respectively. As we can see from the results, a moderate size of band width can provide satisfactory approximation for the LDM as the true density matrix has exponential decay property. In addition, we also conduct quantitative comparisons between algorithm 2 and algorithm 5 in table 1 The relative energy error $\frac{\left|\operatorname{tr}(H P)-\operatorname{tr}\left(H P_{w}\right)\right|}{|\operatorname{tr}(H P)|}$ and the comparable relative differences between $\frac{\left\|P-P_{w}\right\|_{F}}{\|P\|_{F}}$ and $\frac{\left\|\mathfrak{T}_{w}(P)-P_{w}\right\|_{F}}{\|P\|_{F}}$ indicate that results obtained by linear-scaling algorithm 5 can provide satisfactory estimation of the LDM created from algorithm2.

\begin{tabular}{|c|c|c|c|c|c|}
\hline & & $\frac{\left|\operatorname{tr}(H P)-\operatorname{tr}\left(H P_{w}\right)\right|}{|\operatorname{tr}(H P)|}$ & $\frac{\left|\mathscr{E}_{\beta, \eta}(P)-\mathscr{E}_{\beta, \eta}\left(P_{w}\right)\right|}{\left|\mathscr{E}_{\beta, \eta}(P)\right|}$ & $\frac{\left\|P-\mathfrak{T}_{w}(P)\right\|_{F}}{\|P\|_{F}}$ & $\frac{\left\|P-P_{w}\right\|_{F}}{\|P\|_{F}}$ \\
\hline \multirow{3}{*}{$\begin{array}{l}\text { zero temperature } \\
(\beta=\infty, \eta=100, \\
\text { GW) }\end{array}$} & $w=10$ & $1.00 \times 10^{-1}$ & $6.36 \times 10^{-2}$ & $1.46 \times 10^{-1}$ & $2.32 \times 10^{-1}$ \\
\hline & $w=15$ & $4.30 \times 10^{-2}$ & $5.28 \times 10^{-2}$ & $2.24 \times 10^{-2}$ & $3.27 \times 10^{-2}$ \\
\hline & $w=20$ & $4.97 \times 10^{-3}$ & $1.20 \times 10^{-2}$ & $1.01 \times 10^{-3}$ & $1.56 \times 10^{-2}$ \\
\hline \multirow{3}{*}{$\begin{array}{l}\text { finite temperature } \\
(\beta=1, \eta=100, \mathrm{GW})\end{array}$} & $\mathrm{w}=10$ & $8.31 \times 10^{-3}$ & $1.44 \times 10^{-2}$ & $3.86 \times 10^{-3}$ & $8.46 \times 10^{-3}$ \\
\hline & $w=15$ & $5.87 \times 10^{-4}$ & $5.78 \times 10^{-4}$ & $2.51 \times 10^{-4}$ & $5.38 \times 10^{-4}$ \\
\hline & $w=20$ & $9.40 \times 10^{-4}$ & $4.12 \times 10^{-4}$ & $1.36 \times 10^{-4}$ & $3.05 \times 10^{-4}$ \\
\hline \multirow{3}{*}{$\begin{array}{l}\text { finite temperature } \\
(\beta=1, \eta=100 \text {, } \\
\text { potential free })\end{array}$} & $\mathrm{w}=10$ & $2.13 \times 10^{-3}$ & $2.78 \times 10^{-2}$ & $4.04 \times 10^{-3}$ & $5.99 \times 10^{-3}$ \\
\hline & $w=15$ & $3.03 \times 10^{-6}$ & $1.03 \times 10^{-3}$ & $2.66 \times 10^{-4}$ & $4.81 \times 10^{-4}$ \\
\hline & $w=20$ & $1.42 \times 10^{-5}$ & $6.82 \times 10^{-4}$ & $1.54 \times 10^{-4}$ & $2.35 \times 10^{-4}$ \\
\hline
\end{tabular}

TABLE 1. Approximation error using banded matrices with $\eta=100$ for all cases.

In the third experiment, we numerically test the dependence of the matrix band width proposed in the linear scaling algorithms to the $\ell_{1}$ penalty parameter $\eta$ used in the LDMs. As we can observe from the model (11) and the model [12), the parameter $\eta$ controls the strength of the $\ell_{1}$ penalty term. In other words, smaller $\eta$ enforces larger $\ell_{1}$ penalization and leads to more localized behavior of the resulting density matrices, which suggests to use narrower banded matrices to obtain satisfactory approximation results. In Figure 6 the left columns illustrate numerical results for LDMs with the GW potential at zero temperature using $\eta=1,10,100$. As comparisons, the corresponding numerical results using banded matrices ( $w=15,20,25$ respectively) with linear-scaling algorithms are illustrated in the right columns of Figure 6. Similarly, Figure 7illustrates dependence of $w$ to $\eta$ for LDMs with the GW potential at the finite temperature $(\beta=1)$. 

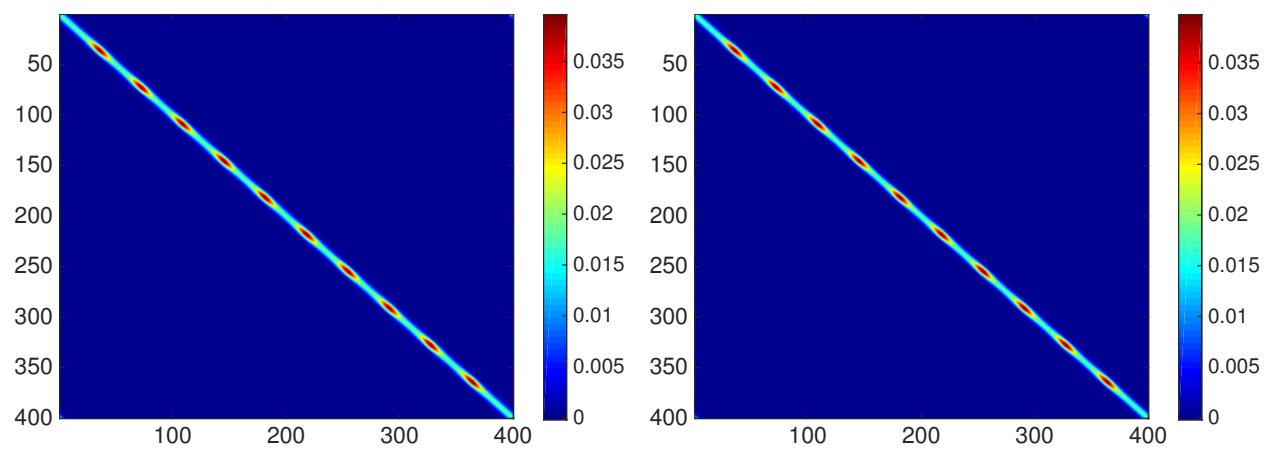

(a)

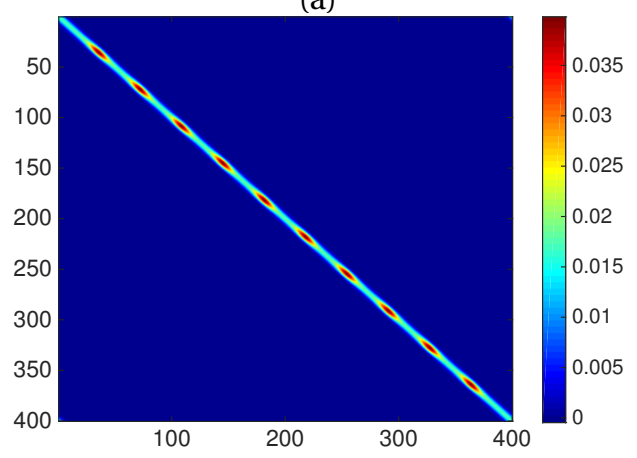

(c)

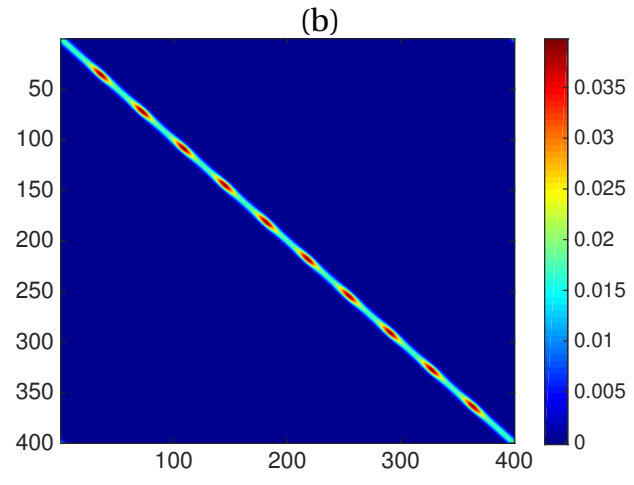

(d)

FIGURE 4. Result comparisons of the finite temperature case with GW potential ( $n=400, N=10, \beta=1$ ). (a). The true density function obtained by the first $\mathrm{N}$ eigenfunctions of $H$. (b). Solution of the localized density matrix with $\eta=100$ using algorithm 2. (c), (d). Solutions of the localized density matrices $(\eta=100)$ using algorithm 5 with $w=10,20$ respectively.

Both figures clearly show that the LDMs obtained from smaller $\eta$ can be approximated well using banded matrices with smaller band width.

In the fourth experiment, we test the computation cost of the proposed algorithms for approximating the eigenvalue thresholding and the Fermi-Dirac operation. Using different matrix sizes, the EigenThresviaChebyPoly designed by algorithm 3 is applied to approximate the step of hard thresholding eigenvalues used in the algorithm 1 step 5 . Similarly, we also test FermiDiracviaChebyPoly for different matrix sizes for the FermiDirac operation. Red curves in Figure $8(a, b)$ report log-log curves of the average computation cost for both cases with respect to different matrix sizes, where blue curves illustrate the corresponding linear fitting for the computation cost curve. For banded matrices, It is clear that the computation costs for both approximation algorithms based on Chebyshev polynomials are linearly scaling to the matrix sizes. 


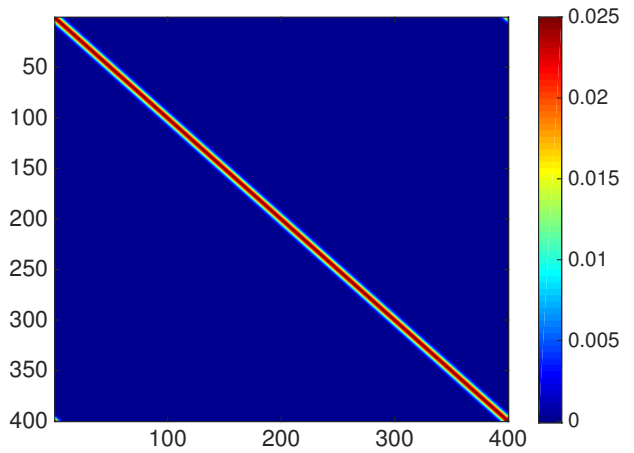

(a)

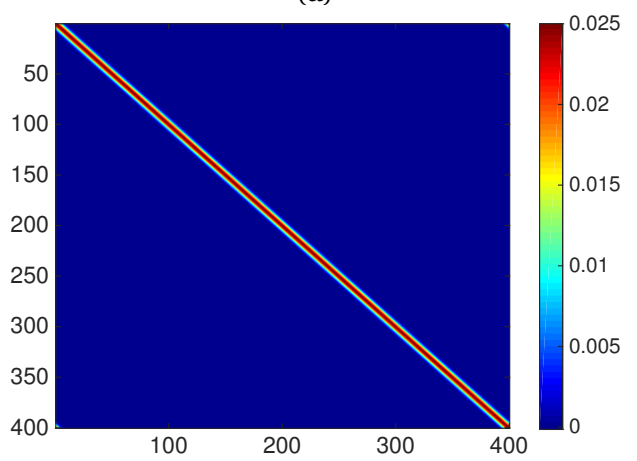

(c)

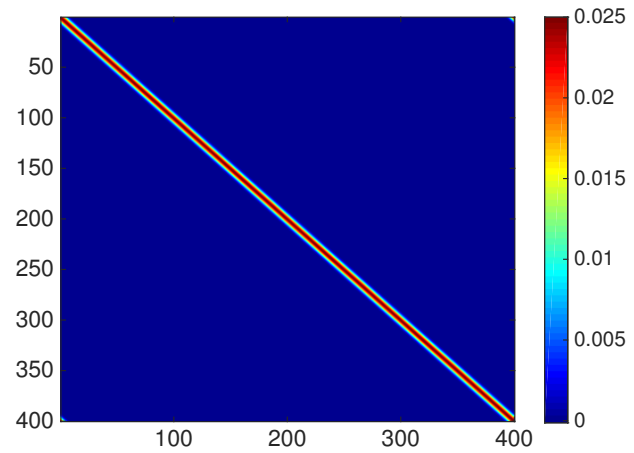

(b)

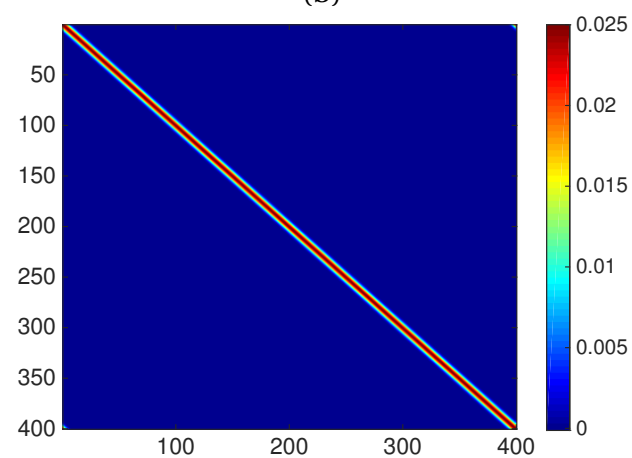

(d)

FIGURE 5. Result comparisons of the potential free finite temperature case $(n=400, N=10, \beta=1)$. (a). The true density function obtained by the first $\mathrm{N}$ eigenfunctions of $H$. (b). Solution of the localized density matrix with $\eta=100$ using algorithm 2 (c), (d). Solutions of the localized density matrices $(\eta=100)$ using algorithm 5 with $w=10,20$ respectively.

We next report comparisons for the computation cost of all proposed algorithms, where we set parameters the same as those used in the first and the second experiments. We first conduct comparison between algorithm 1 and algorithm 4 for the zero temperature case with matrix size chosen from $10^{3}$ to $10^{4}$. Figure 9 (a) reports the log-log plots of average time consumption of each iteration for both algorithms. From the slope of the corresponding linear fitting curves, it is clear to see that the original proposed algorithm 1 has computation cost cubically dependent on the matrix size, while the algorithm 4 based on the banded structure has computation cost linearly dependent on the matrix size. As we can observe from Figure 9 (a), the linear-scaling algorithm can significantly reduce the computation time when $n$ is larger than certain moderate size. Similarly, we also conduct comparison between algorithm 2 and algorithm 5 for the finite temperature case, where the matrix size has been chosen from $10^{2}$ to $2.5 \times 10^{3}$. The slopes of 

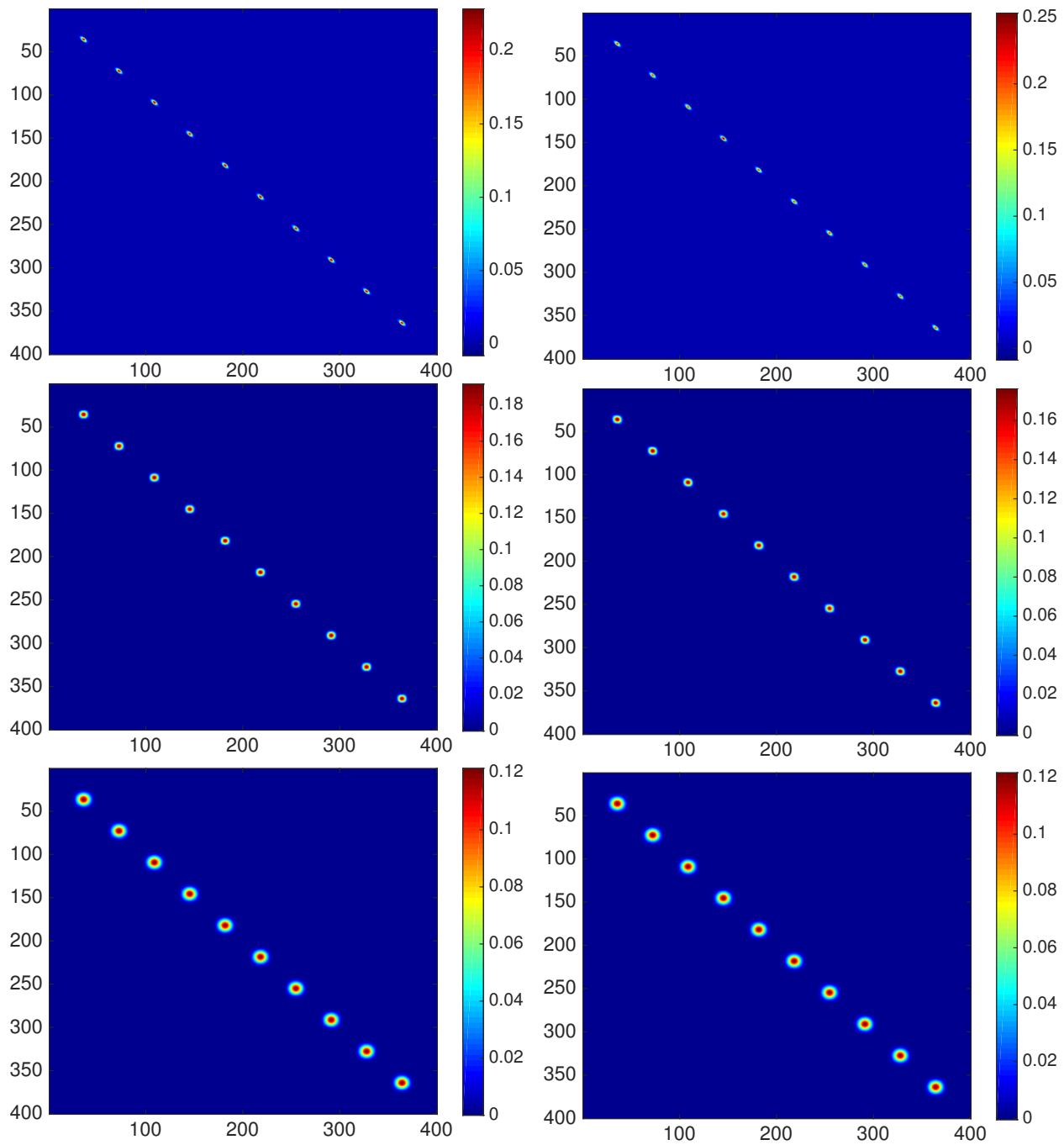

FiguRE 6 . Dependence of the matrix band $w$ and the $\ell_{1}$ penalty parameter $\eta$ of the zero temperature case with the GW potential $(n=$ $400, N=10$ ). Left column (top to bottom): Solution of the localized density matrix with $\eta=1,10,100$ using algorithm 1 Right column (top to bottom): Corresponding solutions of the localized density matrices using algorithm 4 with $m_{e t}=50$ and $(\eta, w)=(1,15),(10,20),(100,25)$ respectively.

the corresponding linear fitting curves also indicate that the proposed algorithm 5 has linear scalelinear-scaling dependent on the problem size, while the original algorithm 

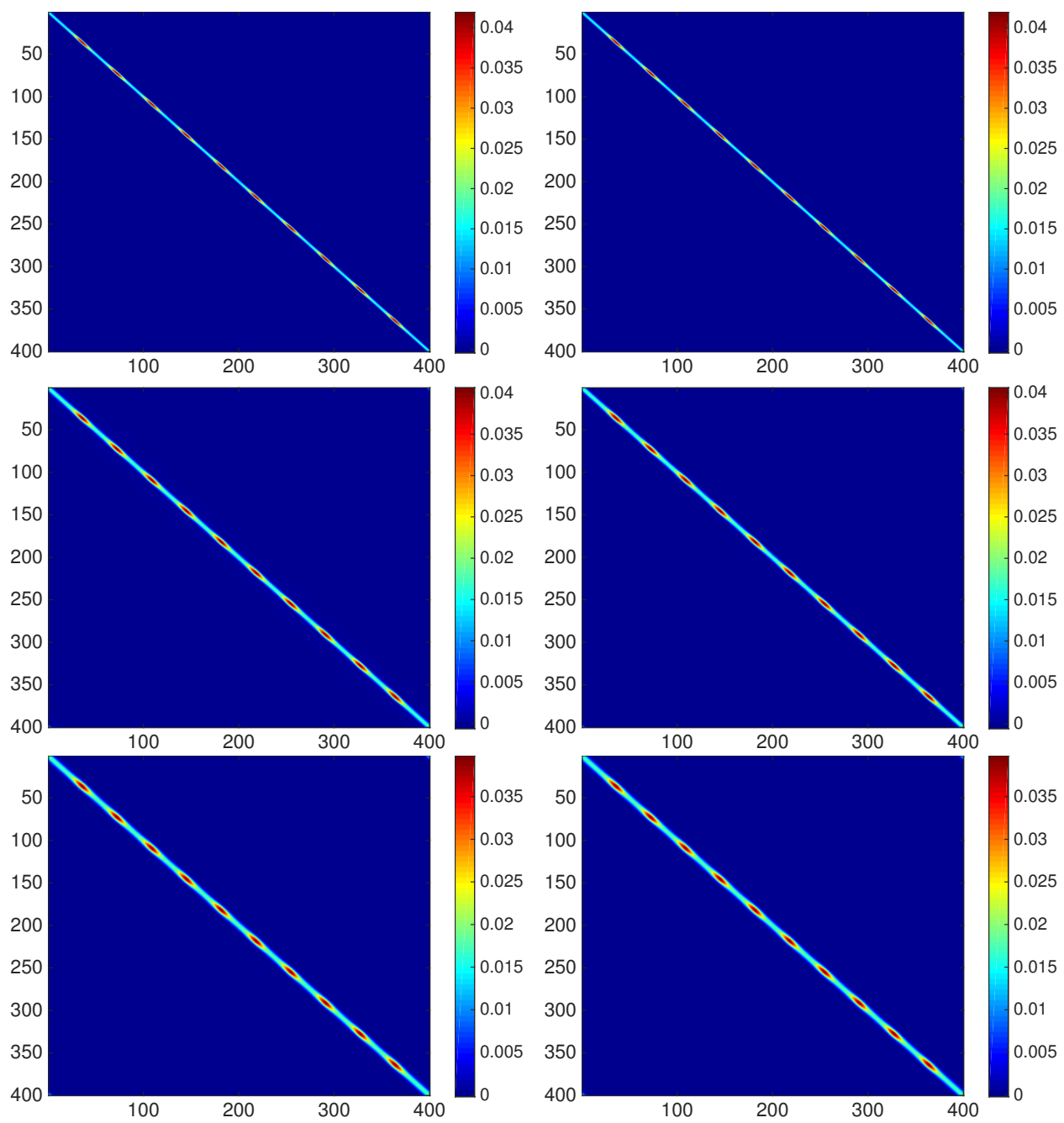

FIGURE 7. Dependence of the matrix band width $w$ to the $\ell_{1}$ penalty parameter $\eta$ of the finite temperature case with the GW potential ( $n=$ $400, N=10, \beta=1$ ). Left column (top to bottom): Solution of the localized density matrix with $\eta=1,10,100$ using algorithm2. Right column (top to bottom): Corresponding solutions of the localized density matrices using algorithm 5 with $m_{e t}=40, m_{f d}=20$ and $(\eta, w)=$ $(1,15),(10,20),(100,25)$ respectively.

2 is cubically scaling to the problem size. Therefore, we can take the linearly scaling advantage of the proposed algorithm 4 and algorithm 5 based on the banded structure for problems with large size. 


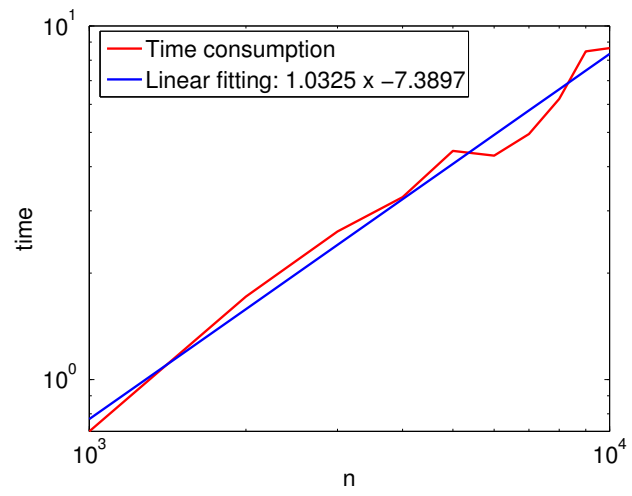

(a)

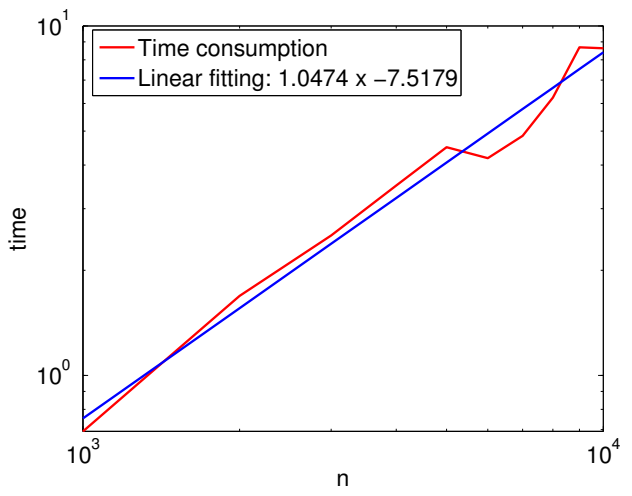

(b)

FIgURE 8. (a): Time consumption of EigenThresviaChebyPoly $(P, 40)$ with different sizes of matrix $P$ and fixed band width $w=10$. (b): Time consumption of FermiDiracviaChebyPoly $(P, 40)$ with different sizes of matrix $P$ and fixed band width $w=10$.

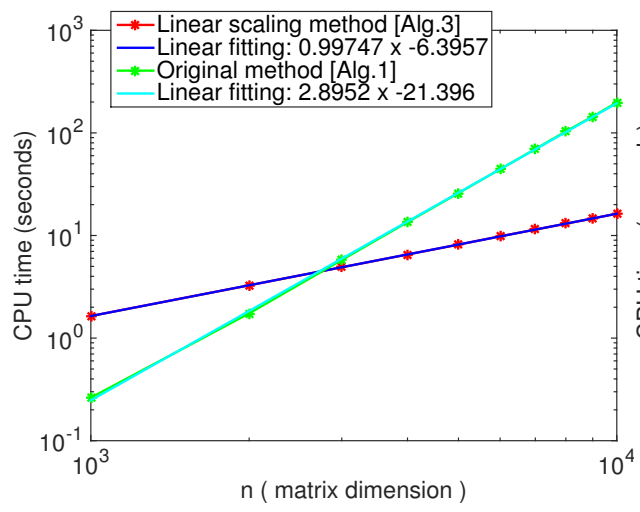

(a)

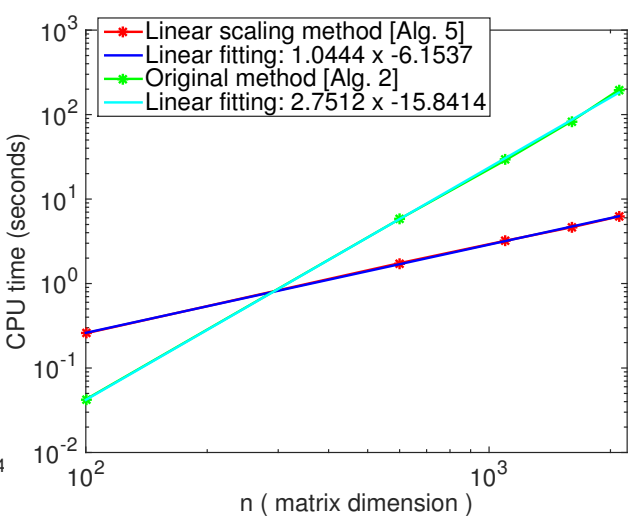

(b)

FIGURE 9. (a): Zero temperature case: time consumption of each iteration used in Alg. 1 v.s. Alg. 4 (b): Finite temperature case: time consumption of each iteration used in Alg. 2 v.s. Alg. 5

\section{CONCLUSION AND FUTURE WORKS}

This work extends our previous work 16 for constructing LDMs to the finite temperature case by adding an $\ell_{1}$ regularization to the energy of the original quantum system. As it has been shown that the density matrix decays exponential away from the diagonal for insulating system or system at finite temperature, the LDMs obtained by the 
proposed convex variational models provide good approximation to the original density matrices. Theoretically, we have conducted analysis to the approximation behavior. In addition, we also design convergence guaranteed numerical algorithms to solve the proposed localized density matrix models based on Bregman iteration. More importantly, by observing that the $\ell_{1}$ regularized system can naturally create a localized density matrix with banded structure, we design approximate algorithms to find the localized density matrices. These numerical algorithms for banded matrices have computation complexity linearly scaling to the matrix size $n$.

This paper mainly focuses on proposing variational methods for LDMs, theoretically analyzing its properties and designing numerical algorithms. Thus, we only test some simple examples in the experimental part to illustrate the proposed modes and algorithms. In particular, we have taken a naive finite difference discretization. All codes are implemented in MATLAB for test purpose and are not optimized. In our future work, we will further accelerate the computation from several aspects. First, we can discretize the system using better localized bases to reduce the problem size 2 | 18 , 30. Second, we can improve the implementation for better computation performance. Moreover, it may be of interest to explore other minimization strategies, for example, combining our localization approaches with the proximal gradient method recently proposed in [32]. In future works, we plan also to explore theoretical analysis for decay properties of the LDM.

\section{REFERENCES}

[1] M. Benzi, P. Boito, and N. Razouk, Decay properties of spectral projectors with applications to electronic structure, SIAM Rev. 55 (2013), 3-64.

[2] V. Blum, R. Gehrke, F. Hanke, P. Havu, V. Havu, X. Ren, K. Reuter, and M. Scheffler, Ab initio molecular simulations with numeric atom-centered orbitals, Comput. Phys. Commun. 180 (2009), no. 11, 2175-2196.

[3] D. R. Bowler and T. Miyazaki, $O(N)$ methods in electronic structure calculations, Rep. Prog. Phys. 75 (2012), 036503.

[4] M. Challacombe, A simplified density matrix minimization for linear scaling self-consistent field theory, J. Chem. Phys. 110 (1999), $2332-2342$.

[5] P. L. Combettes and J.-C. Pesquet, Proximal splitting methods in signal processing, Fixed-point algorithms for inverse problems in science and engineering, 2011, pp. 185-212.

[6] M. S. Daw, Model for energetics of solids based on the density matrix, Phys. Rev. B 47 (1993), 10895.

[7] W. E and J. Lu, The electronic structure of smoothly deformed crystals: Wannier functions and the CauchyBorn rule, Arch. Ration. Mech. Anal. 199 (2011), 407-433.

[8] E. Esser, Applications of Lagrangian-based alternating direction methods and connections to split Bregman, UCLA CAM Report (09-31) (2009).

[9] R. L. Frank, C. Hainzl, R. Seiringer, and J. P. Solovej, Microscopic derivation of Ginzburg-Landau theory, J. Amer. Math. Soc. 25 (2012), 667-713.

[10] C. J. Garcia-Cervera, J. Lu, Y. Xuan, and W. E, A linear scaling subspace iteration algorithm with optimally localized non-orthogonal wave functions for kohn-sham denisty functional theory, Phys. Rev. B 79 (2009), 115110.

[11] S. Goedecker, Linear scaling electronic structure methods, Rev. Mod. Phys. 71 (1999), 1085-1123. 
[12] S. Goedecker and L. Colombo, Efficient linear scaling algorithm for tight-binding molecular dynamics, Phys. Rev. Lett. 73 (1994), 122-125.

[13] T. Goldstein and S. Osher, The split Bregman method for L1-regularized problems, SIAM Journal on Imaging Sciences 2 (2009), no. 2, 323-343.

[14] C. Hainzl, M. Lewin, and R. Seiringer, A nonlinear model for relativistic electrons at positive temperature, Rev. Math. Phys. 20 (2008), 1283-1307.

[15] W. Kohn, Density functional and density matrix method scaling linearly with the number of atoms, Phys. Rev. Lett. 76 (1996), 3168-3171.

[16] R. Lai, J. Lu, and S. Osher, Density matrix minimization with $\ell_{1}$ regularization, Commun. Math. Sci. (in press).

[17] X.-P. Li, R. W. Nunes, and D. Vanderbilt, Density-matrix electronic-structure method with linear systemsize scaling, Phys. Rev. B 47 (1993), 10891-10894.

[18] L. Lin, J. Lu, L. Ying, and W. E, Adaptive local basis functions for Kohn-Sham density functional theory in a discontinuous Galerkin framework I: Total energy calculation, J. Comput. Phys. 231 (2012), 2140-2154.

[19] R. Martin, Electronic structure: basic theory and practical methods, Cambridge University Press, Cambridge, 2004.

[20] D. A. Mazziotti, Towards idempotent reduced density matrices via particle-hole duality: McWeeny's purifcation and beyond, Phys. Rev. E 68 (2003), 066701.

[21] R. McWeeny, Some recent advances in density matrix theory, Rev. Mod. Phys. 32 (1960), 335-369.

[22] N. D. Mermin, Thermal properties of the inhomogeneous electron gas, Phys. Rev. 137 (1965), A1441 -A1443.

[23] A. M. N. Niklasson, Expansion algorithm for the density matrix, Phys. Rev. B 66 (2002), 155115.

[24] A. M. N. Niklasson, Density matrix methods in linear scaling electronic structure theory, Linear-Scaling Techniques in Computational Chemistry and Physics, Challenges and Advances in Computational Chemistry and Physics, vol. 13, 2011, pp. 439-473.

[25] S. Osher, M. Burger, D. Goldfarb, J. Xu, and W. Yin, An iterative regularizatoin method for total variationbased image restoration, Multiscale Model. Simul. 4 (2005), 460-489.

[26] V. Ozolins, R. Lai, R. Caflisch, and S. Osher, Compressed modes for variational problems in mathematics and physics, Prol. Natl. Acad. Sci. USA 110 (2013), 18368-18373.

[27] A. H. R. Palser and D. E. Manolopoulos, Canonical purification of the density matrix in electronic-structure theory, Phys. Rev. B 58 (1998), 12704.

[28] R. Parr and W. Yang, Density-functional theory of atoms and molecules, International Series of Monographs on Chemistry, Oxford University Press, New York, 1989.

[29] D. Ruelle, Statistical mechanics: Rigorous results, Imperial College Press, London and World Scientific, Singapore, 1999.

[30] J. M. Soler, E. Artacho, J. D. Gale, A. Garcia, J. Junquera, P. Ordejón, and D. Sáchez-Portal, The SIESTA method for ab initio order-N materials simulation, J. Phys.: Condens. Matter 14 (2007), no. 11, 2745-2779.

[31] L. N. Trefethen, Approximation theory and approximation practice, SIAM, 2013.

[32] M. Ulbrich, Z. Wen, C. Yang, D. Klöckner, and Z. Lu, A proximal gradient method for ensemble density functional theory, SIAM J. Sci. Comput. 37 (2015), no. 4, A1975-A2002.

[33] C. Wu and X. Tai, Augmented lagrangian method, dual methods and split-bregman iterations for ROF, vectorial TV and higher order models, SIAM J. Imaging Science 3 (2010), no. 3, 300-339.

[34] W. Yin and S. Osher, Error forgetting of bregman iteration, Journal of Scientific Computing 54 (2013), no. 2-3, 684-695.

[35] W. Yin, S. Osher, D. Goldfarb, and J. Darbon, Bregman iterative algorithms for l1-minimization with applications to compressed sensing, SIAM Journal on Imaging Sciences 1 (2008), no. 1, 143-168. 
Department of Mathematics, Rensselaer Polytechnic Institute.

E-mail address: lair@rpi.edu

Departments of Mathematics, Physics, and Chemistry, Duke University

E-mail address: j ianfeng@math.duke.edu 
LaTeX Source Files

s

(1)

(1)

(20)

(2)

(1)

(1)

(1)
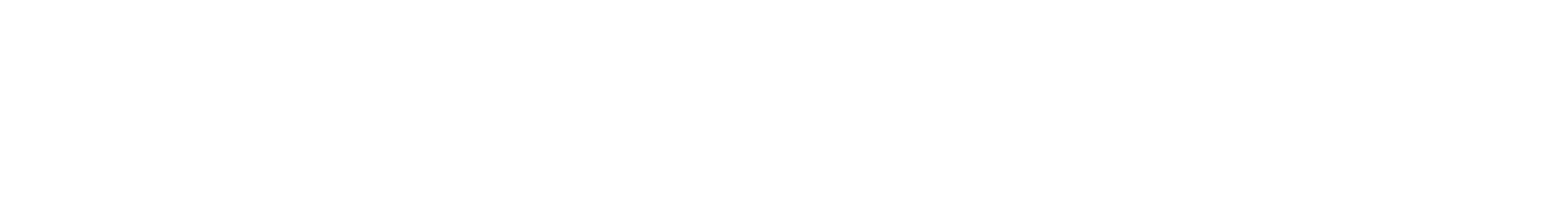

.

.

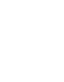

.

.

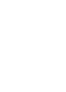

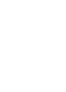

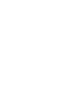

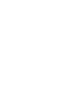

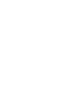

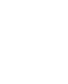

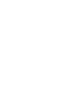

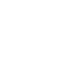

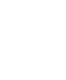

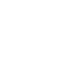

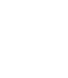

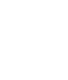

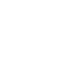

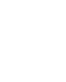

.

(1)

.

.

(

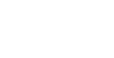

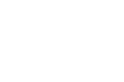

- 